\title{
Synchronization waves in geometric networks
}

\author{
I. Leyva, ${ }^{1,2}$ A. Navas, ${ }^{3}$ I. Sendiña-Nadal, ${ }^{1,2}$ J. M. Buldú,,${ }^{1,2}$ J. A. Almendral, ${ }^{1,2}$ and S. Boccaletti ${ }^{3}$ \\ ${ }^{1}$ Complex Systems Group, Rey Juan Carlos University, Móstoles E-28999, Madrid, Spain \\ ${ }^{2}$ Laboratory of Biological Networks, Center for Biomedical Technology, E-28223 Pozuelo de Alarcón, Madrid, Spain \\ ${ }^{3}$ Group of Computational Systems Biology, Center for Biomedical Technology, E-28223 Pozuelo de Alarcón, Madrid, Spain
}

(Received 7 September 2011; published 6 December 2011)

\begin{abstract}
We report synchronization of networked excitable nodes embedded in a metric space, where the connectivity properties are mostly determined by the distance between units. Such a high clustered structure, combined with the lack of long-range connections, prevents full synchronization and yields instead the emergence of synchronization waves. We show that this regime is optimal for information transmission through the system, as it enhances the options of reconstructing the topology from the dynamics. Measurements of topological and functional centralities reveal that the wave-synchronization state allows detection of the most structurally relevant nodes from a single observation of the dynamics, without any a priori information on the model equations ruling the evolution of the ensemble.
\end{abstract}

DOI: 10.1103/PhysRevE.84.065101

PACS number(s): 89.75.Fb, 87.19.lq, 87.19.1m, 89.75.Kd

In recent years, an increasing number of real systems have found reliable representations as networks of interacting dynamical units [1]. In many cases, information on the networking system can be obtained by only accounting for nodes and interactions between them, disregarding the Euclidean position of its components. Such is the case of the majority of social networks, the World-Wide Web (WWW), and food webs [2]. Nevertheless, there are other relevant cases, such as power grids, Internet routers, epidemics, and neural connectivity, where it is unavoidable to explicitly account for a network's structure embedded into a geometric space, as such a constraint on the spatial positions of the graph's units greatly influences all local and global properties of the graph. Only very recent studies have considered space-dependent network generative models to explain the origin and features of the observed connectivity structure $[3,4]$, with a specific focus on biological neural networks [5].

Furthermore, to what extent a spatial embedding might influence the setting of a collective network's dynamics is a subject attracting much attention in recent research. In particular, some studies have focused on the influence of geometry over the dynamical stability of networks of neurons, as well as over the formation of modules and the network's synchronizability [6,7]. Very recently, critical phenomena and the related appearance of activity avalanches in neural tissues have been hypothesized in experiments as the main mechanisms through which information is transmitted and processed in the graph [8]. Criticality turns out to be of special relevance for revealing the underlying network's structure: In Ref. [9], it was shown that it leads to the reconstruction of the network topology from the observation of the dynamics, after extensive statistics. This is a promising result as, in general, the connectivity structure of a graph cannot be fully revealed only from the observation of its dynamics, and it has to be expected that the spatial embedding, when relevant, could provide additional information to recover properly the topology of a network from dynamical data.

In this paper, we consider the interplay between structure and dynamics in a spatial network of excitable units. In particular, we focus on the graph's coherence properties and study the onset of synchronization waves as a hallmark imprinted by the spatially embedded topology, thus constituting a transitional configuration between synchronization of a complex network and pattern formation in continuous extended media. We show that, in association with such a waving regime, there is a strong correlation between the functional and topological centralities that helps in capturing the most topologically relevant nodes, without any previous knowledge of the network growth model.

Let us start with generating the network spatial configuration. To this end, we randomly seed $N=500$ nodes in a two-dimensional Euclidean space of size $L \times L$, with $L=100$. We further consider that the probability of node $i$ of establishing a link with node $j$ of the ensemble is given by the distance-dependent rule $p(r)=p_{0} e^{-\left(\frac{r}{\sigma}\right)^{2}}$, where $p_{0}$ is a constant, $r \equiv d_{i j}$ is the Euclidean distance between nodes $i$ and $j$ in the plane, and $\sigma$ is a parameter regulating the link outreach [4]. For large values of $\sigma, p(r) \sim p_{0}$ and the resulting network becomes space independent, equivalent to an Erdös-Renyi random configuration. In contrast, small values of $\sigma$ generate networks that lead to a strong clustering and highly modular structure. The procedure is similar to that of random geometric graphs (RGGs) [10], despitethe fact that the link between two nodes relies on probability rather than linking volume. Links are added here until a target mean degree $\langle k\rangle$ is obtained, and the resulting graphs are tested to feature a single connected component of size $N$. As the node density is $N / L^{2}$ [and $p(r \rightarrow 2 \sigma) \rightarrow 0$ ], the mean target degree is $\langle k\rangle_{\max } \sim \frac{N}{L^{2}} \pi(2 \sigma)^{2}$, which gives $\langle k\rangle_{\max }=15$ for $\sigma=5$, which is the minimum value of $\sigma$ below which it is not warranted that all nodes have at least one connection.

While the scenario that will be described holds qualitatively for a generic excitable system, for the sake of illustration we use the case in which the node $i$ is a neuron unit, obeying the Morris-Lecar model [11,12]:

$$
\begin{aligned}
C \dot{V}_{i}= & I_{i}^{\mathrm{ext}}-g_{C a} M_{\infty}\left(V_{i}\right)\left(V_{i}-V_{C a}\right)-g_{K} W_{i}\left(V_{i}-V_{K}\right) \\
& -g_{\mathrm{L}}\left(V_{i}-V_{\mathrm{L}}\right)-\frac{d}{k_{i}} \sum_{j}^{N} a_{i j}\left(V_{i}-V_{j}\right)
\end{aligned}
$$




$$
\dot{W}_{i}=\frac{W_{\infty}\left(V_{i}\right)-W_{i}}{\tau\left(V_{i}\right)} .
$$

Here $V_{i}$ is the soma potential, $W_{i}\left(V_{i}\right)$ is the fraction of open $\mathrm{K}^{+}$channels, and $W_{\infty}\left(V_{i}\right)\left[M_{\infty}\left(V_{i}\right)\right]$ stands for the $K^{+}\left(\mathrm{Ca}^{+2}\right)$ channel voltage-dependent saturation value in the absence of stimulation. $I_{i}^{\text {ext }}$ is the external current source, and the three addends account for the internal ionic $\mathrm{Ca}^{+2}, \mathrm{~K}^{+}$, and leakage currents, with their corresponding channel conductivities $g_{\mathrm{Ca}}$, $g_{\mathrm{K}}$, and $g_{\mathrm{L}}$. The model parameters are selected so as to produce class II neural excitability with a bifurcation point at $I_{i}^{\text {ext }}=I^{\text {th }}=0.33$, signaling a transition from an excitable to an oscillatory regime as $I^{\text {ext }}$ is increased. Each neuron is injected with an external current $I_{i}^{\text {ext }}=I_{i}^{0}+\varepsilon_{i}$, being $I_{i}^{0}$ randomly distributed within the interval $(0.32 \pm 0.2)$, and $\varepsilon_{i}$ a Gaussian white noise of zero mean and standard deviation 0.1 , uncorrelated for each node. As a consequence, part of the nodes are above the spiking threshold $I^{\text {th }}$, and the system exhibits a spontaneous activity whose spatiotemporal features are the object of our study. $\mathbf{A}=\left\{a_{i j}\right\}$ is the connectivity adjacency matrix, $d$ is the coupling strength, and $k_{i}$ is the number of connections of node $i$.

In order to quantify the synchronization in a way convenient for experimentalists, we calculate the coherence between nodes $i$ and $j$ as the correlation between the the time series of spikes at zero delay, within a time bin $\Delta t$. To that end, the system is evolved for a long time $T$. Then, this interval is divided in $\Gamma$ small time bins of size $\Delta t$ (i.e., $T=\Gamma \Delta t$ ). Each spiking sequence is then converted into a binary series $B_{i}$ by assigning the value $B_{i}(\tau)=1$ if node $i$ has spiked within the $\tau^{\text {th }}$ bin, and 0 otherwise, for $\tau=1,2, \ldots, \Gamma$. Finally, the quantity $s_{i j}$ is introduced to account for the dynamical coherence between nodes $i$ and $j$ [13]:

$$
s_{i j}=\frac{\sum_{\tau=1}^{\Gamma} B_{i}(\tau) B_{j}(\tau)}{\sqrt{\sum_{\tau=1}^{\Gamma} B_{i}(\tau) \sum_{\tau=1}^{\Gamma} B_{j}(\tau)}} .
$$

This way, the global network synchronization $S$ can be calculated as the ensemble average of the coherence matrix $\mathbf{S}=\left\{s_{i j}\right\}$. Figure 1(a) reports the value of $S$ versus $\sigma$ and $d$ for $\langle k\rangle=15$. As it is seen, the system can only reach significantly high global synchronization for $d$ values over a critical threshold $d_{c} \sim 0.08$. However, even at high $d>d_{c}$, small values of $\sigma$ prevent the system from reaching global synchronization. This is of particular relevance in biological networks, where often full synchronization is associated with a pathological state [14]: For instance, the excess of longrange connectivity in some cortex areas is considered as the main hypothesis for epileptic seizures. To gain a deeper insight into the loss of global synchrony within this region, we define the local synchronization as $S_{\rho}=\left\langle s_{i j}\right\rangle, d_{i j}<\rho$, where $\rho$ is a synchronization radius. Figure 1(b) shows the behavior of $S_{\rho}$ in the $[d, \sigma]$ phase space. We can observe that the threshold value of $d$ required for the onset of local synchronization is similar to that obtained for the global synchrony. Nevertheless, a significant difference arises when comparing both synchronization indicators, since local synchrony is maintained even for moderate values of $\sigma$. This fact indicates that the influence of long-range connections is much less important locally than globally. In Fig. 1(c), we plot (a)
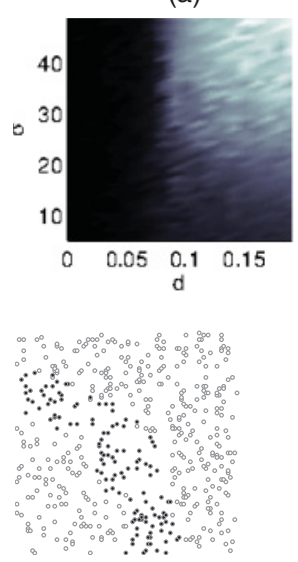

(b)
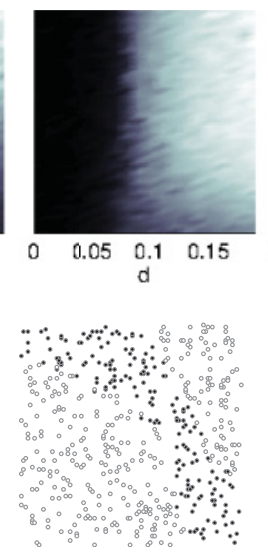

(c)

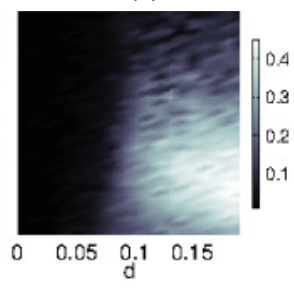

FIG. 1. (Color online) (a) Global synchronization $S$, (b) local synchronization $S_{\rho}$, and (c) wave synchronization $S_{w}=S_{\rho}-S$ (see text for definitions) in the parameter space $(d, \sigma)$. In all cases, $\rho=10$ and $N=500$. The color code is reported in the bar at the right of the three plots. (d) Successive snapshots of the activity of the network, for $\langle k\rangle=15, d=0.2$ and $\sigma=10$. Empty (full) circles correspond to inactive (spiking) nodes. The underlying network has been hidden for a better visualization.

the difference between both measures, $S_{w}=S_{\rho}-S$, showing the region where the network locally synchronizes despite not featuring a global coherent behavior. Interestingly, this region is characterized by the appearance of synchronization waves [15]. For a coupling strength $d>d_{c}$, we can observe how the wave dynamics is affected by $\sigma$. The system departs from an asynchronous regime (low values of both $S_{\rho}$ and $S$ ) and enters a region where the width of the wave fronts increases with $\sigma$, eventually collapsing into a full synchronization regime for sufficiently high values of $\sigma$, that is, once the spatial constraint progressively loses its influence. Therefore, the difference $S_{w}=S_{\rho}-S$ is a good indicator of the existence of a wave regime. An example of a synchronization wave is reported in Fig. 1(d), where we plot three successive snapshots of a wave front propagating through the network at $\sigma=10$ and $d=0.2$. The nonlocal coupling makes this waving phenomenon different in many aspects from waves observed in continuous excitable media [16] as, for example, the facts that wave-front widths depend on the long-range coupling outreach and that propagation of coherent spatial structures like spiral waves is hindered by the shortcut links between nodes [17].

Notice that such a wave dynamics is not present in nongeometric networks, where the route to synchronization occurs instead via clustering processes for the random case (ER) or evolves from hubs in scale free (SF) [18,19]. This difference can be observed in Fig. 2, where we consider carefully the synchronization edge as a function of $d$ for a small $\sigma$ value ( $\sigma=10$, at which the maximum of $S_{w}$ occurs) and a very large one ( $\sigma=1000$, for which the resulting network is identical to a nongeometrical ER graph). As discussed previously, the full synchronization is only reachable for large $\sigma$ [Fig. 2(a)], where for $\sigma=10$ the system remains very far from global synchronization. Below $d_{c}$, the global synchronization is indistinguishable in both cases, but in Fig. 2(b) we can observe how the different link outreach 

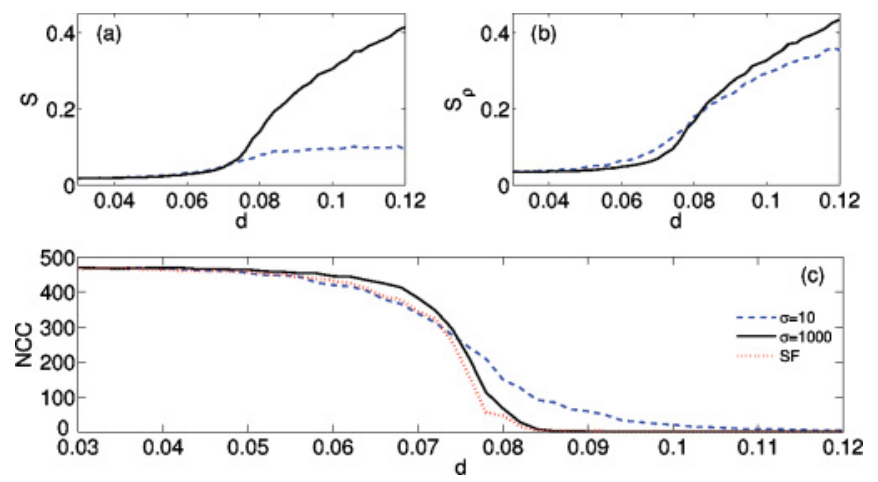

FIG. 2. (Color online) Synchronization route as a function of the coupling strength $d$ for $\sigma=10$ (blue dashed lines) and $\sigma=$ 1000 (black continuous lines). (a) Global synchronization $S$ and (b) local synchronization $S_{\rho}$. (c) Number of synchronized connected components for $\sigma=10, \sigma=1000$, and scale-free network (red dotted lines).

yields to the a different evolution of the radial synchronization $S_{\rho}$ : The high clustering of the $\sigma=10$ network helps to create synchronized components that, in this case, roughly correspond to geometrical neighbors. This group grows faster than in the ER case, coinciding with the development of the wave. This is clear in Fig. 2(c), representing the number of connected components of the $\mathbf{S}$ matrix as a function of $d$ for $\sigma=10, \sigma=1000$, and SF configuration networks. Here, in order to understand $\mathbf{S}$ as an adjacency matrix, we use a threshold $T$ such that $s_{i j}=1$ if $s_{i j}>T$, and $s_{i j}=0$ otherwise. The number of connected components is the multiplicity of the null eigenvalue of the Laplacian matrix obtained from the resulting binary synchronization matrix. It can be seen as the local synchronization for small $\sigma$ behaves in a way similar to a SF network for small $d$, since the synchronized component grows from the most clustered areas, acting as a hubs, up to form the wave front. As $d$ increases, the wave gets defined, and the number of connected components decreases smoothly as the wave sharpens.

The wave-propagation regime, where the information transmission through the system seems to be maximal [8], is an optimal state to explore the relationship between dynamics and topology. The recovering of information on the network structure from measurements of the network dynamics is a relevant issue that has been considered in literature [20], particularly in the case of brain dynamics where the topic has generated much interest [12,21].

In the present case, we demonstrate that the wave passage generates a real-time functional enlightening of the underlying network structure. To this end, we calculate the time-dependent centrality correlation $C_{c}(\tau)=\operatorname{corr}\left[\vec{C}_{S}(\tau), \vec{C}_{A}\right]$ for each time bin $\tau$. Here, $\vec{C}_{A}$ is the eigenvector centrality of the adjacency matrix $\mathbf{A}$, while $\vec{C}_{S}(\tau)$ is the eigenvector centrality calculated for the instantaneous coherence matrix $\mathbf{S}(\tau)$ whose elements are $s_{i j}(\tau)=B_{i}(\tau) B_{j}(\tau)$. In Fig. 3 we show the value of $C_{c}(\tau)$ and the global synchronization inside each bin $S(\tau)=\langle\mathbf{S}(\tau)\rangle$, along with the raster plot, for the same values of $N,\langle k\rangle$, and $d$ and two values of $\sigma(\sigma=10$, corresponding to wave propagation, and $\sigma=30$, closer to full synchronization). In Figs. 3(a) and 3(b), the dynamics corresponds to a wave
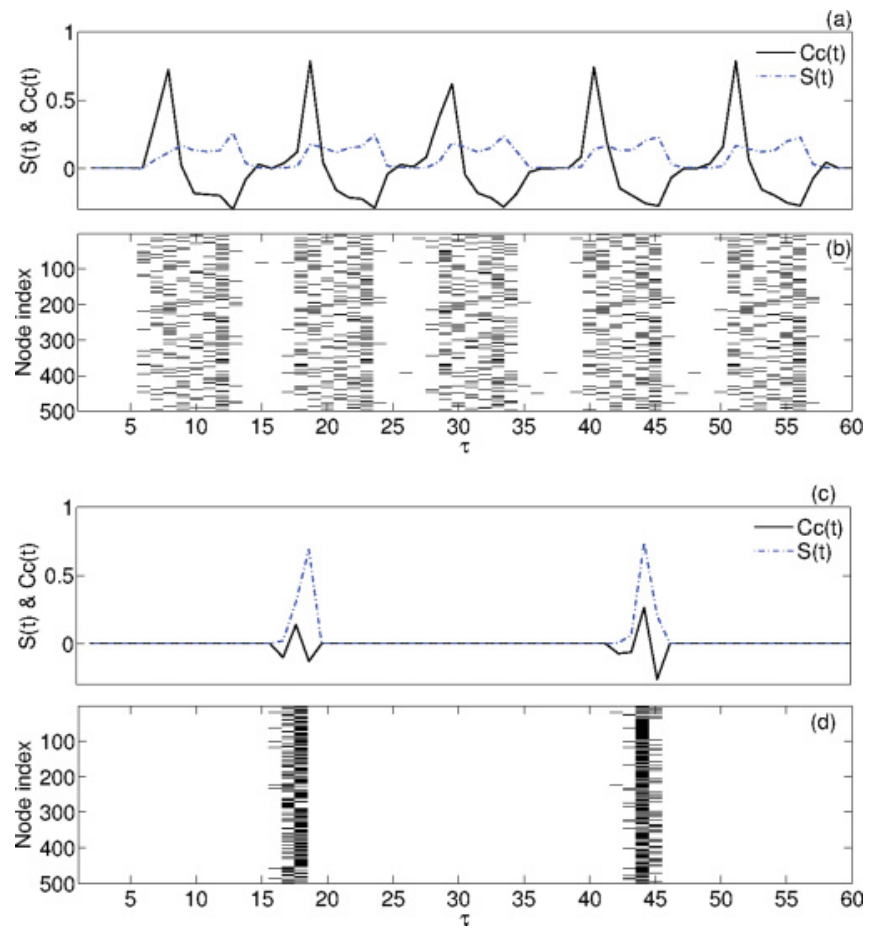

FIG. 3. (Color online) (a), (c) Temporal evolution of the centrality correlation $C_{c}(\tau)$ (black solid line) and global synchronization at each bin $S(\tau)$ (blue dash-dotted line). (b), (d) Raster plots of the network activity. See text for the definition of all quantities. Parameters are $N=500,\langle k\rangle=15, d=0.2$, and [(a), (b)] $\sigma=10$ and [(c), (d) $\sigma=30$.

propagation where, as we have seen, global synchronization (dotted line) is not very high. Nevertheless, during the wave passage, the running value of $C_{c}(\tau)$ (full line) reaches a maximum close to 0.8 , revealing a strong bounding between structural and functional centralities. In contrast, for fully developed synchronization [Figs. 3(c) and 3(d)], the correlation $C_{c}(\tau)$ nearly vanishes, showing small fluctuations only when nodes are activated.

The reported results suggest, therefore, that the regime of wave propagation is an optimal candidate for the task of recovering the structure of the network from the observation of its dynamics. In fact, the knowledge of the relationship between the most functionally relevant areas and their (a)

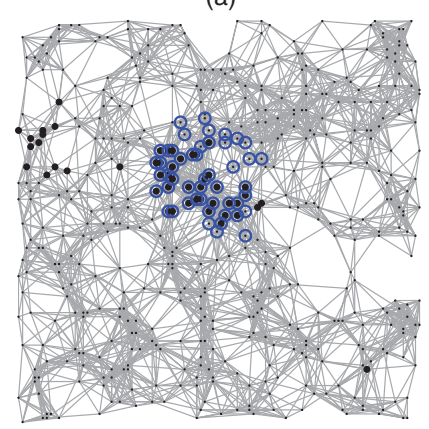

(b)

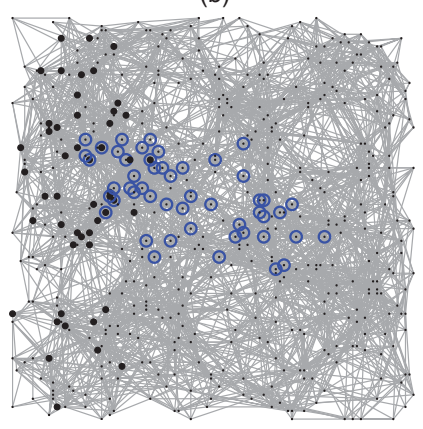

FIG. 4. (Color online) Sample nets showing the 50 nodes with the highest topological (o) and functional $(\bullet)$ centrality for (a) $\sigma=10$ and (b) $\sigma=25$. 
underlying structures is enough to give insight into the whole network. In Fig. 4, we plot some examples of the 50 nodes with the highest topological $(\bullet)$ and functional (०) centrality for $d=0.2$ in two distinct situations: the wave-propagation regime at $\sigma=10$ depicted in Fig. 4(a) and a close to fully synchronized regime at $\sigma=25$ in Fig. 4(b). The comparison clearly shows that the prediction of the node roles is strikingly good in the wave-synchronization regime, while the relationship between structure and dynamics becomes weaker and weaker as the level of global synchronization grows in the network.

In conclusion, we have studied the properties of synchronization of a geometric complex network, where the nodes are embedded in real space and the link probability decreases with the Euclidean distance. We have shown that a high density of connections with close neighbors combined with the lack of long-range connections prevents full synchronization, and it allows the emergence of synchronization waves. This latter state strongly correlates the topological and functional centralities of nodes, thus enhancing the option of inferring the network topology from the observation of the dynamics, up to the point of allowing detection of the central areas from only functional coherence data. Our results, therefore, can be of relevance in all circumstances where one has full access to functional measurements and the possibility of a direct inspection of the topological structure (i.e., the probing of the underlying connectivity matrix) is prevented as, for instance, is often the case in biology.

This work was supported by the Spanish Ministry of Science and Technology [FIS2009-07072] and by the Community of Madrid under Project URJC-CM-2010-CET-5006 and the research and development program MODELICO-CM [S2009ESP-1691].
[1] S. Boccaletti, V. Latora, Y. Moreno, M. Chavez, and D. U. Hwang, Phys. Rep. 424, 175 (2006).

[2] M. E. J. Newman, SIAM Review 45, 167 (2003).

[3] A. F. Rozenfeld, R. Cohen, D. Ben-Avraham, and S. Havlin, Phys. Rev. Lett. 89, 218701 (2002); M. Zanin, J. M. Buldú, P. Cano, and S. Boccaletti, Chaos 18, 023103 (2008); J. Billen, M. Wilson, A. Baljon, and A. Rabinovitch, Phys. Rev. E 80, 046116 (2009); M. Barthélemy, Phys. Rep. 499, 1 (2011).

[4] M. Kaiser and C. C. Hilgetag, Phys. Rev. E 69, 036103 (2004); S. Itzkovitz and U. Alon, ibid. 71, 026117 (2005).

[5] A. Pérez-Escudero and G. G. de Polavieja, PNAS 104, 17180 (2007); S. Itzkovitz, L. Baruch, E. Shapiro, and E. Segal, ibid. 105, 9278 (2008); J. P. Onella, S. Arbesman, M. C. González, A. L. Barabasi, and N. Christakis, PLoS One 6, e16939 (2011); M. Kaiser, Neuroimage 57, 892 (2011).

[6] C. M. Schneider-Mizell, J. M. Parent, E. Ben-Jacob, M. R. Zochowski, and L. Sander, Phys. Bio. 7, 046008 (2010); R. Segev, M. Benveniste, Y. Shapira, and E. Ben-Jacob, Phys. Rev. Lett. 90, 168101 (2003); C. Zhou, L. Zemanova, G. Zamora, C. C. Hilgetag, and J. Kurths, ibid. 97, 238103 (2006).

[7] A. Diaz-Guilera, J. Gomez-Gardeñes, Y. Moreno, and M. Nekovee, Int. J. Bifurcation Chaos Appl. Sci. Eng. 19, 687 (2009).

[8] J. M. Beggs, Phil. Trans. R. Soc. A 366, 329 (2008); S. Bradde, F. Caccioli, L. Dall'Asta, and G. Bianconi, Phys. Rev. Lett. 104, 218701 (2010).
[9] S. Pajevic and P. Plenz, PLoS Comput. Biol. 5, e1000271 (2009). [10] J. Dall and M. Christensen, Phys. Rev. E 66, 016121 (2002).

[11] C. Morris and H. Lecar, Biophys. J. 35, 193 (1981).

[12] E. Fuchs, A. Ayali, E. Ben-Jacob, and S. Boccaletti, Phys. Bio. 6, 036018 (2009).

[13] S. Kochubey and L. Savtchenko, Neurophysiology 40, 224 (2008).

[14] K. Lehnertz, S. Bialonski, M.-T. Horstmann, D. Krug, A. Rothkegel, M. Staniek, and T. Wagner, J. Neurosci. Methods 183, 42 (2009); T. J. Netoff, R. Clewey, T. Keck, and J. A. White, J. Neurosci. 24, 8075 (2004).

[15] O.-U. Kheowan, E. Mihaliuk, B. Blasius, I. Sendiña-Nadal, and K. Showalter, Phys. Rev. Lett. 98, 074101 (2007).

[16] V. Casagrande, Y. Togashi, and A. S. Mikhailov, Phys. Rev. Lett. 99, 048301 (2007).

[17] Q. Wanga, M. Perc, Z. Duana, and G. Chen, Phys. Lett. A 372, 5681 (2008).

[18] A. Arenas, A. Diaz-Guilera, J. Kurths, Y. Moreno, and C. Zhou, Phys. Rep. 469, 93 (2008).

[19] J. Gómez-Gardeñes, Y. Moreno, and A. Arenas, Phys. Rev. Lett. 98, 034101 (2007).

[20] J. Zhang, C. Zhou, X. Xu, and M. Small, Phys. Rev. E 82, 026116 (2010); S. G. Shandilya and M. Timme, New J. Phys. 13, 013004 (2011).

[21] S. Feldt, J. X. Wang, E. Shtrahman, R. Dzakpasu, E. Olariu, and M. Zochowski, Phys. Bio 7, 046004 (2010). 